\section{4}

In addition to the striking chemical diversity and the structural flexibility, the organic composition allows for additional functionalities that are impossible in their inorganic counterparts

\title{
Purely organic perovskites
}

Ferroelectric materials exhibit spontaneous electric polarization in the absence of an external electric field. Upon application of an electric field, this polarization switches, and upon removal of the field, the polarization is retained. As a consequence, ferroelectric materials are avidly sought after for many applications, including memory devices, capacitors and sensors. However, ferroelectrics typically contain metals and can be costly and toxic, as well as requiring high processing temperatures.

Inorganic perovskites and a handful of hybrid inorganic-organic perovskites show ferroelectric behaviour; however, purely organic perovskites with this behaviour have proven elusive. The advantages that organic perovskite ferroelectrics could offer make them an attractive synthetic goal.
Now, Yu-Meng You, Ren-Gen Xiong and colleagues report a family of metal-free, organic perovskites that are ferroelectric at room temperature. The organic perovskites have the general formula $\mathrm{ABX}_{3}$, where $\mathrm{A}$ is a divalent organic cation, $\mathrm{B}$ is $\left(\mathrm{NH}_{4}\right)$ and $\mathrm{X}$ is $\mathrm{Cl}, \mathrm{Br}$ or I. The perovskite structures are 3D networks of corner-shared $\left(\mathrm{NH}_{4}\right) \mathrm{X}_{6}$ octahedra with divalent organic ammonium cations inside the octahedral cavities.

The researchers synthesised 23 members of this family of organic perovskites and identified their best performing ferroelectric material as $\mathrm{MDABCO}-\mathrm{NH}_{4} \mathrm{I}_{3}(\mathrm{MDABCO}=$ $N$-methyl- $N^{\prime}$-diazabicyclo[2.2.2] octonium) with a spontaneous polarization $\left(P_{\mathrm{S}}\right)$ of $22 \mu \mathrm{C} \mathrm{cm}^{-2}$. This ferroelectric performance is close to that of barium titanate,

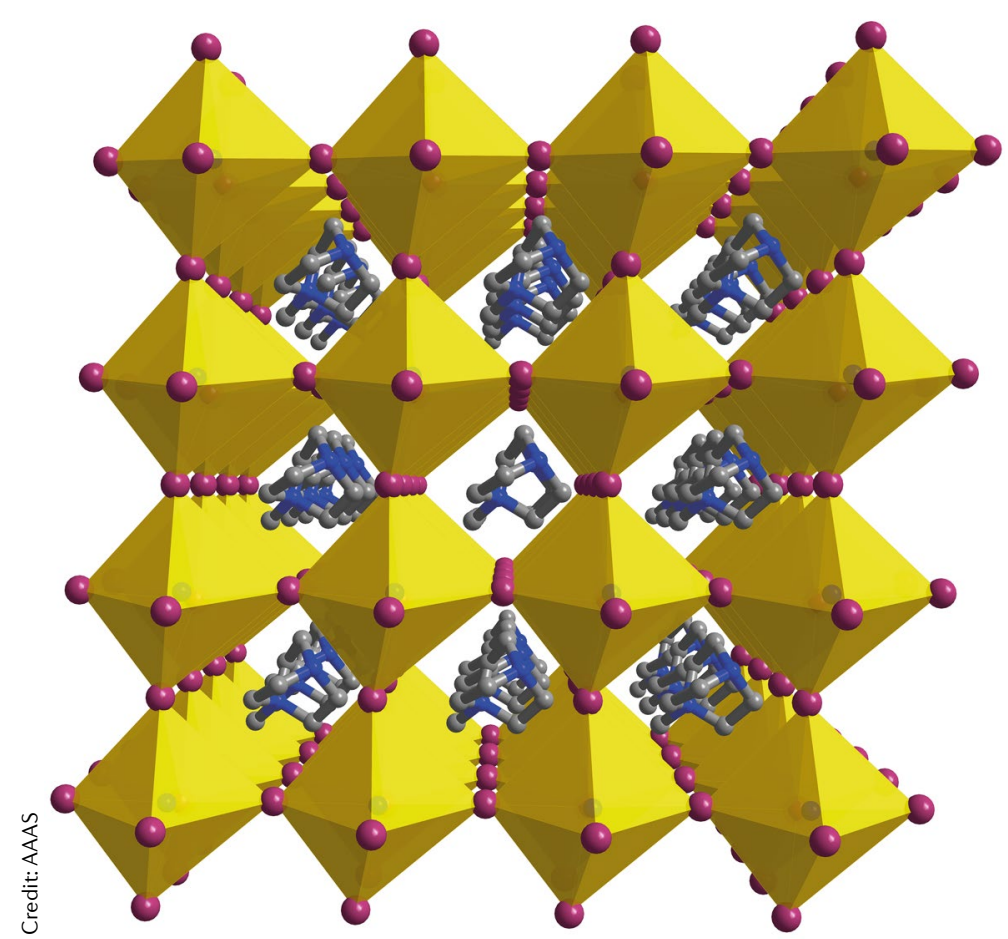

an inorganic perovskite, and the phase transition temperature (to the paraelectric phase) at $448 \mathrm{~K}$ is higher than the phase transition of barium titanate.

The addition of a methyl group to the 'spherical' cation, DABCO, lowers the molecular symmetry and equips the resultant cations with dipole moments that align to give spontaneous polarization. However, the quasispherical shape of the cation ensures molecular reorientation is possible. "At higher temperature, the organic cations exhibit severely dynamic disorder and are modelled as a spherical structure," explains Xiong. "The transition of the organic cation from a static state to a nearly freely rotating state results in the high-symmetric paraelectric phase."

"In addition to the striking chemical diversity and the structural flexibility, the organic composition allows for additional functionalities that are impossible in their inorganic counterparts, such are chiral centres," says Xiong. In the future, these homochiral ferroelectric metal-free perovskites could be used as electro-optical devices, such as optically active polarizing switches or in chiral resolution or chiral catalysis experiments in the pharmaceutical and biological industries.

"The large $P_{\mathrm{s}}$, the visible thermal hysteresis and the striking entropy change accompanying the paraelectric-to-ferroelectric phase transition suggests a promising future for these organic compositions as electrocaloric materials in solid-state cooling devices," concludes Xiong.

\section{Alison Stoddart}

